\title{
Ethibond Suture Granuloma Formation Following Repair of Acute Achilles Tendon Ruptures
}

\author{
Amr Darwish ${ }^{1}$, Osama Gamal ${ }^{2}$, Mohamed Kamal Mesregah ${ }^{3}$
}

\begin{abstract}
Aim and objective: Achilles tendon rupture can be adequately treated by surgical repair. This study aimed to represent the incidence, presentation, outcome of treatment of Ethibond granuloma, which is a rare complication following surgical repair.

Materials and methods: This study was a review of 672 patients who had open surgical repair of the Achilles tendon rupture using Ethibond sutures. Eleven (1.6\%) patients developed suture granuloma postoperatively and were included in the study. Epidemiological, clinical, radiological, and histopathological data were reviewed. The outcome of treatment was evaluated.

Results: This study included ten males and one female, with a mean age of $31.4 \pm 13.7$ years. Two patients had diabetes mellitus, and three patients were smoking. All patients presented with swelling and discharge around the surgical site compromising their daily living activities. The mean time interval between the surgical procedure and the diagnosis of granuloma formation was $6 \pm 3.2$ months. Patients were treated by surgical debridement and granuloma excision. The healing and fixation strength of the primary repair were not affected. The wound healed entirely at a mean of $2.6 \pm 0.5$ weeks. With a mean follow-up period of $14.6 \pm 2.2$ months, all patients had a fully functional tendon and were able to resume normal daily living activities.

Conclusion: Ethibond sutures may rarely cause granuloma formation months after the primary repair of Achilles tendon rupture. The granuloma does not affect the strength of the primary repair and can be treated adequately with debridement and excision with a complete wound resolution. Clinical significance: Defining the incidence and outcome of treatment of Ethibond suture granuloma as a devastating complication following acute Achilles tendon repair.

Level of evidence: Level IV, retrospective.

Keywords: Achilles tendon repair, Ethibond granuloma, Foreign body reaction, Granuloma formation, Sinus formation.

Journal of Foot and Ankle Surgery (Asia Pacific) (2021): 10.5005/jp-journals-10040-1148
\end{abstract}

\section{INTRODUCTION}

Achilles tendon is the most frequent lower limb tendon to be acutely ruptured, with an increasing incidence in recent years. ${ }^{1}$ Achilles tendon rupture is more common in males, especially in the $3 \mathrm{rd}$ or 4th decades of their life. ${ }^{2,3}$

The growing popularity of sports and increased participation in them among youth and older people in recent decades may explain the increased incidence of acute Achilles tendon rupture. ${ }^{1,4}$

Some factors were reported to increase the risk of ruptures, which include previous use of anabolic steroids or fluoroquinolone antibiotics, repeated microtrauma, and local injection of corticosteroids. ${ }^{5,6}$

Treatment of acute rupture aims to restore the structural integrity of the Achilles tendon to permit the return to the preinjury level of activity, including participation in sports activities., ${ }^{7,8}$

Surgical treatment of acute Achilles tendon rupture is preferred over conservative management, which is associated with a high risk of re-rupture. ${ }^{3,9,10}$ In contrast, surgical management reduces the rate of re-rupture and allows earlier return to functional activities, but it may be complicated by wound problems such as delayed wound healing, wound breakdown, and necrosis. ${ }^{3,7,11,12}$ The skin overlying the Achilles tendon, through which the longitudinal incision passes, is thin and poorly vascularized, increasing the risk of wound problems. ${ }^{13,14}$

Several non-absorbable suture materials were used for the repair of the Achilles tendon, including PDS II (polydioxanone), Ethibond, ultra-high-molecular-weight polyethylene (UHMWPE), and braided polyethylene terephthalate. ${ }^{15-17}$ The material of the
1,2Department of Orthopaedic Surgery, Faculty of Medicine, Menoufia University, Shebin El-Kom, Menoufia, Egypt

${ }^{3}$ Department of Orthopaedic Surgery, Keck School of Medicine, University of Southern California, Los Angeles, California, USA

Corresponding Author: Mohamed Kamal Mesregah, Department of Orthopaedic Surgery, Keck School of Medicine, University of Southern California, Los Angeles, California, USA, Phone: +1 (213)-952-9585, e-mail: mohamed.mesregah@med.menofia.edu.eg

How to cite this article: Darwish A, Gamal O, Mesregah MK. Ethibond Suture Granuloma Formation Following Repair of Acute Achilles Tendon Ruptures. J Foot Ankle Surg (Asia Pacific) 2021;8(1):12-15.

Source of support: Nil

Conflict of interest: None

suture used for repair can significantly decrease the risk of these wound complications, as well as providing a high-intensity repair. ${ }^{17}$

In the current study, we sought to represent the incidence, presentation, course, and outcome of treatment of Ethibond suture granuloma following repair of acute Achilles tendon rupture.

\section{Materials and Methods}

This study was a review of medical records of 672 consecutive patients who had acute Achilles tendon closed ruptures and were treated by open surgical repair of the Achilles tendon using Ethibond sutures (Ethicon ${ }^{\circledR}$ division of Johnson \& Johnson, Somerville, New Jersey, USA), from January 2013 to May 2019 in a single academically- 
supervised trauma center. Eleven (1.6\%) patients developed suture granuloma in the follow-up period and were included in the study. Our Institutional Review Board approved this study.

Exclusion criteria were open injuries, re-ruptures, history of previous ankle surgeries, and use of suture materials other than Ethibond.

The collected data included age, gender, side of injury, mode of rupture, duration from injury to the initial surgery, the length of the primary incision of the repair, time of appearance of the swelling from the initial surgery, size of the granuloma, management, and follow-up of the suture granuloma. Radiological, laboratory, histopathological investigations, and ankle photographs were also reviewed.

Data analysis was done using IBM SPSS version 20.0 (Armonk, New York, USA: IBM Corp). Categorical variables were reported as frequency counts and percentages, and continuous variables were reported as the median and minimum to maximum range or the mean \pm standard deviation.

\section{Results}

This study identified 11 (1.6\%) of 672 patients who developed a suture granuloma following repair of their Achilles tendon with multifilament, non-absorbable, polyester Ethibond suture material applied using the Krackow technique. This study included ten males and one female, with a mean age of $31.4 \pm 13.7$ (ranging from 5-58) years. Two patients had diabetes mellitus, and three patients were smokers. The involved side was right in seven patients and left in four patients. The mode of injury was sports trauma in ten patients and fall on foot in one patient. The mean time from trauma to the primary surgical repair was $2 \pm 1$ (ranging from 1-4) days. The mean length of the primary incision was $8 \pm 1.7$ (ranging from $5-11) \mathrm{cm}$. All ruptures were repaired using the Krackow technique by Ethibond 2-0 sutures.

All patients presented with swelling and discharge around the surgical site compromising their daily living activities (Fig. 1A). The mean time interval between the surgical procedure and the diagnosis of suture granuloma was $6 \pm 3.2$ (ranging from 1-12) months.

The mean length of the mass was $4.8 \pm 0.5$ (ranging from $4.1-5.4) \mathrm{cm}$, the mean width was $3.9 \pm 0.5$ (ranging from 2.9-4.9) $\mathrm{cm}$, and the mean height was $4.7 \pm 0.8$ (ranging from $3.2-5.9$ ) cm.
In all patients, the mass was detected on the former incision scar with a draining sinus connecting the mass and the skin. Magnetic resonance imaging (MRI) showed granulomatous abscess around the distal portion of the Achilles tendon.

Patients were treated by surgical debridement and granuloma excision (Fig. 1B). The healing and fixtion strength of the primary repair were not affected. Inside the excised mass, an Ethibond suture was detected.

Histopathological reports showed features of foreign body reaction, with giant cells and eosinophilic infiltration. Tissue cultures did not reveal any organisms.

The wound healed entirely at a mean of $2.6 \pm 0.5$ (ranging from 2-3) weeks (Fig. 1C). Patients were followed-up for a mean time of $14.6 \pm 2.2$ (ranging from 12-18) months and had no further complaints (Table 1). All patients had a fully functional tendon, and they were able to resume their normal activities.

\section{Discussion}

Achilles tendon ruptures are usually caused by a sports trauma, with a higher incidence in recent years due to the increased popularity of recreational sports. ${ }^{18}$

Primary surgical repair of acute Achilles tendon rupture has good outcomes; however, postoperative wound complications should be kept in mind. ${ }^{19-21}$

In addition to smoking, steroid use, obesity, and diabetes mellitus, the type of suture materials used for the repair has also been reported to cause wound problems. ${ }^{22-24}$ Maffulli et al. ${ }^{25}$ reported a higher incidence of wound complications in nonathletic patients, which may be explained by a higher prevalence of obesity, hyperlipidemia, diabetes, and smoking in non-athletic individuals.

Non-absorbable sutures are usually used for tendon repairs. The materials of these sutures should be strong enough to withstand forces during healing of the repaired tendon and in the rehabilitation period. ${ }^{16,26}$

In this study, we demonstrated a rare complication of Ethibond suture granuloma leading to a chronic sinus, secondary to repair of Achilles tendon rupture, representing chronic inflammation and deep-seated infection.
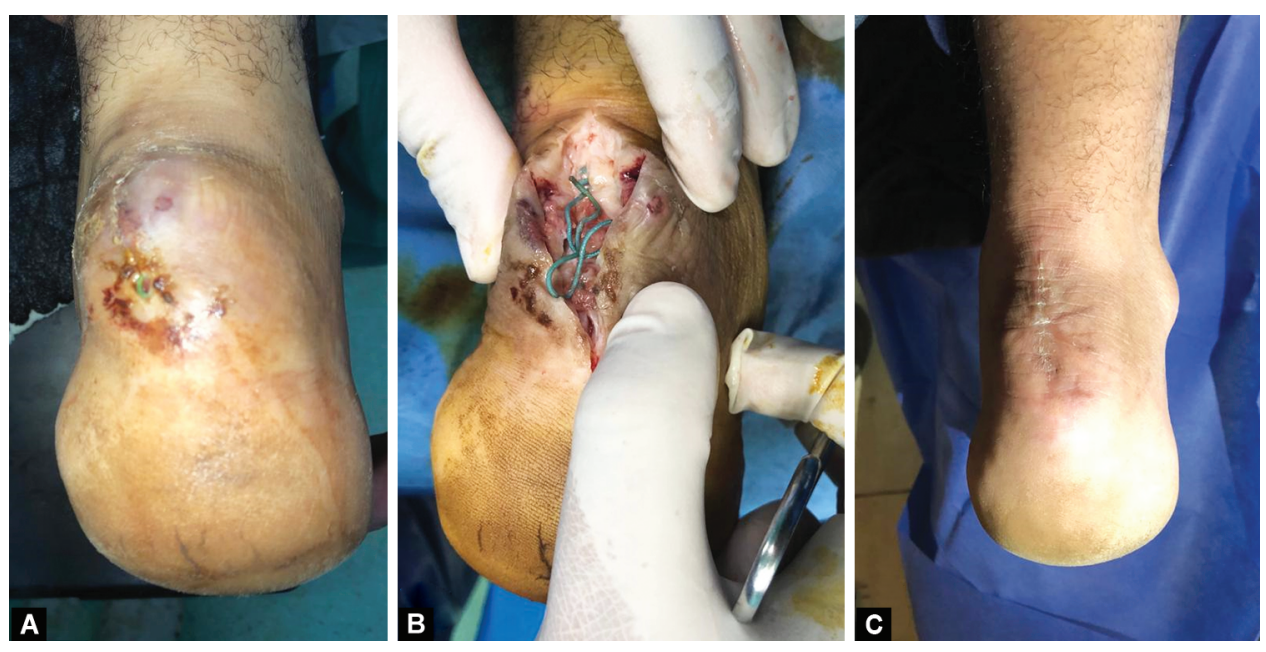

Figs $1 \mathrm{~A}$ to C: A case of male patient 58 years old (No. 2) who developed Ethibond suture granuloma 8 months following repair of acute Achilles tendon repair: (A) Ankle photograph showing swelling and discharging sinus; (B) Intraoperative photograph showing granuloma mass related to Ethibond sutures; (C) Three weeks postoperative follow-up ankle photograph showing complete wound healing 


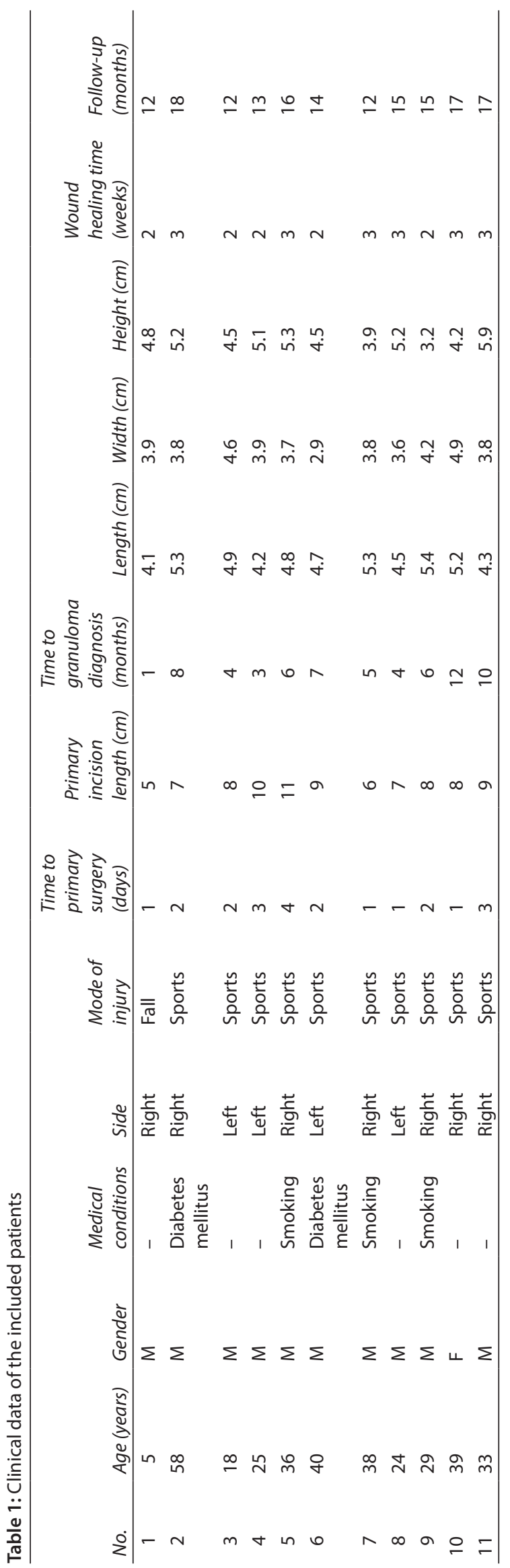

The Ethibond suture can provide a high-strength repair of Achilles tendon rupture; however, it may cause soft tissue problems, including granuloma formation. ${ }^{15,27}$ This possible soft tissue reaction does not affect the healing and fixation strength of the primary repair. ${ }^{15}$ In our study, after excision of the granuloma, the Achilles tendon was found to be intact with high-strength primary repair.

The Ethibond suture is coated with polybutilate, giving it more favorable mechanical characteristics and fewer tissue reactions compared to uncovered braided polyesters. ${ }^{17}$ In a rabbit model study, Esenyel et al. ${ }^{28}$ described a lower inflammatory reaction 6 weeks postoperatively when using Ethibond as compared to other non-absorbable materials.

Foreign body granuloma secondary to Achilles tendon repair with braided polyethylene and polyglactin sutures has been previously reported in the literature. ${ }^{22,24}$ In our study, the incidence of soft tissue granuloma following repair of Achilles tendon rupture by Ethibond sutures was rare with an overall rate of $1.6 \%$.

Ethibond suture granuloma was reported in a few case reports. Kara et al. ${ }^{15}$ reported a case of granulomatous abscess formation 4 months after Achilles tendon repair with Ethibond. The patient was treated with granuloma excision and was followed up for 12 months with no complaints. ${ }^{15}$ Similarly, all patients in our study had no complaints following granuloma excision, after a mean follow-up period of 14.6 months.

Ahluwalia et al. ${ }^{27}$ reported a case of Achilles tendon rupture treated with open repair using modified Kessler's technique with Ethibond suture and developed a deep sinus and chronic infection several months later, which did not respond to antibiotics and surgical debridement. Magnetic resonance imaging demonstrated a sinus tract that was connected to a suture knot, and the suture was removed with full wound healing and no further problem at 3-month follow-up. ${ }^{27}$

Despite being a very rare complication, Ethibond suture granuloma should be suspected in patients who develop erythema, swelling, and discharge months following the repair. This rare complication can be troublesome and should be meticulously treated by debridement and granuloma excision. We would suggest that surgical repair of the Achilles tendon should be carefully performed, giving special attention to burying the core stitch within the tendon substance to reduce the incidence of this granuloma. Careful wound irrigation before skin closure is also crucial. Early MRI is beneficial in detecting an underlying suture abscess. Locating the offending suture and granuloma excision is the key to a successful resolution. The limitations of this study include the small number of patients which is justified by the rare incidence of Ethibond suture granuloma.

\section{Conclusion}

Ethibond sutures provide a high-strength repair for acute Achilles tendon rupture; however, it may rarely cause granuloma formation months after the primary repair, which may be disabling. Ethibond suture granuloma does not affect the strength of the primary repair and can be treated adequately with debridement and excision with a complete resolution and no further complaints.

\section{Clinical Significance}

Defining the incidence and outcome of treatment of Ethibond suture granuloma as a devastating complication following acute Achilles tendon repair. 


\section{References}

1. Fischer S, Colcuc C, Gramlich Y, et al. Prospective randomized clinical trial of open operative, minimally invasive and conservative treatments of acute Achilles tendon tear. Arch Orthop Trauma Surg 2020. DOI: 10.1007/s00402-020-03461-z Online ahead of print.

2. Huttunen TT, Kannus P, Rolf C, et al. Acute Achilles tendon ruptures: incidence of injury and surgery in Sweden between 2001 and 2012. Am J Sports Med 2014;42(10):2419-2423. DOI: 10.1177/0363546514540599.

3. Yongliang $Y$, Honglei J, Wupeng Z, et al. Intraoperative ultrasonography assistance for minimally invasive repair of the acute Achilles tendon rupture. J Orthop Surg Res 2020;15(1):258. DOI: 10.1186/s13018-02001776-6.

4. Lemme NJ, Li NY, DeFroda SF, et al. Epidemiology of Achilles tendon ruptures in the United States: athletic and nonathletic injuries from 2012 to 2016. Orthop J Sports Med 2018;6(11):2325967118808238.

5. Thevendran G, Sarraf KM, Patel NK, et al. The ruptured Achilles tendon: a current overview from biology of rupture to treatment. Musculoskelet Surg 2013;97(1):9-20. DOI: 10.1007/s12306-0130251-6.

6. Spoendlin J, Layton JB, Mundkur M, et al. The risk of Achilles or biceps tendon rupture in new statin users: a propensity score-matched sequential cohort study. Drug Saf 2016;39(12):1229-1237. DOI: 10.1007/ s40264-016-0462-5.

7. Lantto I, Heikkinen J, Flinkkila T, et al. A prospective randomized trial comparing surgical and nonsurgical treatments of acute Achilles tendon ruptures. Am J Sports Med 2016;44(9):2406-2414. DOI: 10.1177/0363546516651060.

8. Haapasalo H, Peltoniemi U, Laine HJ, et al. Treatment of acute Achilles tendon rupture with a standardised protocol. Arch Orthop Trauma Surg 2018;138(8):1089-1096. DOI: 10.1007/s00402-018-2940-y.

9. Wang D, Sandlin MI, Cohen JR, et al. Operative versus nonoperative treatment of acute Achilles tendon rupture: an analysis of 12,570 patients in a large healthcare database. Foot Ankle Surg 2015;21(4):250-253. DOI: 10.1016/j.fas.2015.01.009.

10. Lerch TD, Schwinghammer A, Schmaranzer F, et al. Return to sport and patient satisfaction at 5-year follow-up after nonoperative treatment for acute Achilles tendon rupture. Foot Ankle Int 2020;41(7):784-792. DOI: 10.1177/1071100720919029.

11. Barfod KW, Bencke J, Lauridsen HB, et al. Nonoperative dynamic treatment of acute Achilles tendon rupture: the influence of early weight-bearing on clinical outcome: a blinded, randomized controlled trial. J Bone Joint Surg Am 2014;96(18):1497-1503. DOI: 10.2106/JBJS.M.01273.

12. Willits $K$, Amendola $A$, Bryant $D$, et al. Operative versus nonoperative treatment of acute Achilles tendon ruptures: a multicenter randomized trial using accelerated functional rehabilitation. J Bone Joint Surg Am 2010;92(17):2767-2775. DOI: 10.2106/JBJS.I.01401.

13. Aktas $S$, Kocaoglu B. Open versus minimal invasive repair with Achillon device. Foot Ankle Int 2009;30(5):391-397. DOI: 10.3113/ FAI-2009-0391.
14. Rippstein PF, Jung M, Assal M. Surgical repair of acute Achilles tendon rupture using a "mini-open" technique. Foot Ankle Clin 2002;7(3):611-619. DOI: 10.1016/S1083-7515(02)00040-2.

15. Kara A, Celik H, Seker A, et al. Granuloma formation secondary to Achilles tendon repair with nonabsorbable suture. Int J Surg Case Rep 2014;5(10):720-722. DOI: 10.1016/j.ijscr.2014.07.026.

16. Kocaoglu B, Ulku TK, Gereli A, et al. Evaluation of absorbable and nonabsorbable sutures for repair of Achilles tendon rupture with a suture-guiding device. Foot Ankle Int 2015;36(6):691-695. DOI: $10.1177 / 1071100714568868$.

17. Ji $Y, M a X$, Wang $X$, et al. Different sutures in the surgical treatment of acute closed Achilles tendon rupture. Indian J Surg 2015;77(S3):936940. DOI: 10.1007/s12262-014-1068-8.

18. Chan JJ, Chen KK, Sarker S, et al. Epidemiology of Achilles tendon injuries in collegiate level athletes in the United States. Int Orthop 2020;44(3):585-594. DOI: 10.1007/s00264-019-04471-2.

19. Moller M, Movin T, Granhed H, et al. Acute rupture of tendon Achillis. A prospective randomised study of comparison between surgical and non-surgical treatment. J Bone Joint Surg Br 2001;83(6):843-848. DOI: 10.1302/0301-620X.83B6.0830843.

20. Pajala A, Kangas J, Ohtonen $P$, et al. Rerupture and deep infection following treatment of total Achilles tendon rupture. J Bone Joint Surg Am 2002;84(11):2016-2021. DOI: 10.2106/00004623-20021100000017.

21. Dalton GP, Wapner KL, Hecht PJ. Complications of Achilles and posterior tibial tendon surgeries. Clin Orthop Relat Res 2001. 133-139. DOI: 10.1097/00003086-200110000-00014.

22. Bekler HI, Beyzadeoglu T, Gokce A, et al. Aseptic drainage associated with polyglactine sutures used for repair of Achilles tendon ruptures. Acta Orthop Traumatol Turc 2008;42(2):135-138. DOI: 10.3944/ AOTT.2008.42.2.135

23. Bruggeman NB, Turner NS, Dahm DL, et al. Wound complications after open Achilles tendon repair: an analysis of risk factors. Clin Orthop Relat Res 2004. 63-66. DOI: 10.1097/01.blo.0000144475.05543.e7.

24. Ollivere BJ, Bosman HA, Bearcroft PW, et al. Foreign body granulomatous reaction associated with polyethelene 'Fiberwire((R))' suture material used in Achilles tendon repair. Foot Ankle Surg 2014;20(2):e27-e29. DOI: 10.1016/j.fas.2014.01.006.

25. Maffulli N, Testa V, Capasso G, et al. Surgery for chronic Achilles tendinopathy yields worse results in nonathletic patients. Clin J Sport Med 2006;16(2):123-128. DOI: 10.1097/00042752-200603000-00007.

26. Rupp S, Georg T, Gauss C, et al. Fatigue testing of suture anchors. Am J Sports Med 2002;30(2):239-247. DOI: 10.1177/03635465020300021601.

27. Ahluwalia R, Zourelidis C, Guo S, et al. Chronic sinus formation using non absorbable braided suture following open repair of Achilles tendon. Foot Ankle Surg 2013;19(2):e7-e9. DOI: 10.1016/j. fas.2012.11.003.

28. Esenyel CZ, Demirhan M, Kilicoglu O, et al. Evaluation of soft tissue reactions to three nonabsorbable suture materials in a rabbit model. Acta Orthop Traumatol Turc 2009;43(4):366-372. DOI: 10.3944/ AOTT.2009.366. 